\title{
Notas sobre medicina y difusión de ideas en la Grecia clásica
}

\author{
César SierRa MARTíN \\ Universitat Autònoma de Barcelona (Proyecto RYC2010-05622) \\ cesar.sierra@e-campus.uab.cat
}

Recibido: 14-11-2011

Aceptado: 25-11-2011

\section{RESUMEN}

En el presente artículo ponemos de manifiesto la importancia de la medicina en la difusión de ideas sobre la naturaleza del hombre. En este sentido, la conexión entre medicina y retórica junto a la movilidad que tenían los médicos nos induce a pensar que ciertas ideas recogidas en los Tratados hipocráticos debieron ser ampliamente conocidas en la época clásica.

Palabras clave: Naturaleza del hombre, medicina itinerante, corpus hipocrático.

\begin{abstract}
The aim of this paper is to underline the importance of medicine in the dissemination of ideas about human being. The connection between medicine and rethoric with physicians' mobility lead us to think that certain hippocratic ideas should be widely disseminated in classical Greece.
\end{abstract}

Key words: Human being, traveling medicine, Hippocratic corpus.

\section{DEBATIENDO LA NATURALEZA DEL HOMBRE}

Entre finales del siglo vi a.C. y la primera mitad del v a.C. cobró importancia en el

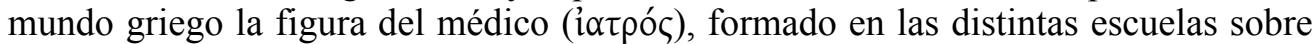
medicina, destacando las de Cnido, Cos, Cirene, Crotona y Rodas ${ }^{1}$. Estos profesionales se caracterizaron por tratar de combatir la enfermedad (en jonio voũ $\sigma \circ$ ), investigando sus causas y comprendiendo la naturaleza humana ( $\varphi$ v́øı)). Para realizar dichas pesquisas de forma sistemática, los médicos aplicaron un método de investigación (iotopín) basado en la observación y el racionalismo². Dicho de otra forma, los médicos racionales (pre-hipocráticos e hipocráticos) comenzaron a entender la naturaleza

${ }^{1}$ Laín (1970: 403), López Férez (1986: 159).

${ }^{2}$ La aplicación médica de la iơopín puede verse en Sobre la medicina antigua 20. También debe tenerse en cuenta los análisis de Jouanna (1992: 93 y ss); Lara Nava (2006: 20 y ss). 
humana a partir de evidencias perceptibles que dieron lugar a la elaboración de teorías sobre la salud. En este sentido, destacamos la figura de Alcmeón de Crotona (s. VI a.C.) que desarrolló el concepto de «isonomía», equilibrio de cualidades en el cuerpo, y «supremacía», cuando alguna de estas cualidades (seco, húmedo, caliente, amargo, dulce...) se imponía al resto y producía un desequilibrio, provocando la enferme$\mathrm{dad}^{3}$. De manera análoga entendieron el cuerpo humano los médicos hipocráticos

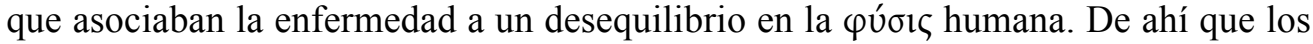

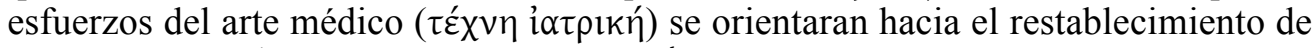

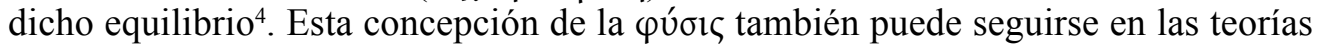
del filósofo Empédocles de Agrigento, que defendía la existencia de una pluralidad de principios o raíces (fuego, aire, agua y tierra) las cuales, en equilibrio, configuraban el mundo ${ }^{5}$. Así, los precursores de esta medicina racional plantearon una «fisiología» basada en la contraposición de elementos o fuerzas opuestas (enantíosis) que regían el cuerpo humano y conformaban un equilibrio definitorio de la salud ${ }^{6}$. Este planteamiento señala que las fuerzas que regían el universo no eran diferentes a las que gobernaban el cuerpo humano, estableciendo una relación entre cosmología y medicina que puede observarse al inicio de los tratados Sobre el viento, Aires, aguas

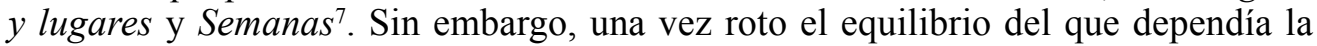
salud, la curación se abordaba utilizando el diálogo con el paciente y la exploración sensorial. Estos elementos proporcionaban al médico las evidencias necesarias para emitir un diagnóstico o juicio sobre su estado ${ }^{8}$. A partir de aquí el médico decidía el tratamiento a seguir en función de la naturaleza de la dolencia. Por ejemplo, si el médico interpretaba que la causa de la enfermedad se debía al estilo de vida ( $\delta i ́ \alpha \imath \tau \alpha)$, podía prescribir una dieta específica combinada con ejercicios, todo ello orientado a restablecer la salud del paciente 9 .

Sin embargo, esta idea contravenía otras posturas desarrolladas por filósofos presocráticos, y también médicos, que entendían la naturaleza a partir de un único principio activo $^{10}$. Es el caso de Tales de Mileto quien, a principios del vi a.C. señaló al agua como sustancia originaria del mundo. Anaxímenes, en la segunda mitad del VI a.C, opinaba que era el aire. Heráclito de Éfeso, a finales del vi a.C., indicó que dicho ele-

${ }^{3}$ Las ideas de Alcmeón se recogen en (Aëtius, V 30, 1 D.K.24B4); comentado en Longrigg (1993: 47-51).

4 Laín (1970: 65). Recientemente Kosak (2004: 30 y ss.) realiza un completo análisis de las fuentes médicas que razonan sobre el arte médico. Schiefsky (2005: 25 y ss.), hace lo propio en Sobre la medicina antigua.

${ }^{5}$ Véase, por ejemplo, Arist. Met. 984a; Simpl., Fís. 33, 18; Aëtius, I. 3, 20. Laín (1987: 165) y Lloyd (1999: 35 y ss.), relacionan al filósofo Empédocles con el tratado Sobre la enfermedad sagrada (20) y con otras ideas contemporáneas sobre el hombre. En general sobre Empédocles véase Graham 1999.

${ }^{6}$ Laín (1970: 80).

${ }^{7}$ En definitiva, una relación entre el hombre y el universo (Jouanna 1988: 25 y ss.).

${ }^{8}$ Laín (1976: 109-111).

${ }^{9}$ La dietética, entendida como un estilo de vida que englobaba la alimentación, el ejercicio y el trabajo, era una disciplina muy difundida en la Grecia clásica y una pieza central de la medicina hipocrática. Mediante un correcto estilo de vida se podía preservar el equilibrio natural de la salud; Joly (1967: XI-XII).

${ }^{10}$ Los denominados monistas; Cano-Cuenca (2003: 21). Por ejemplo en (Sobre los vientos IV. 1), la causa de todas las enfermedades es el viento, que rige en todos los cuerpos del universo, Jouanna (1988: 11). 
mento era el fuego, mientras que Parménides de Elea, a principios del v a.C., defendió la unidad, uniformidad e inmovilidad del $\operatorname{ser}^{11}$. No es este el lugar para el desarrollo en profundidad de estas ideas filosóficas pero sí queremos destacar la complejidad del debate intelectual en el que intentaban abrirse paso los médicos.

Así, los médicos hipocráticos del siglo v a.C. debían tener una idea clara sobre la phýsis humana para desmarcarse de intelectuales que trataban sobre esa misma cuestión pero en otros términos ${ }^{12}$. Los tratados hipocráticos ${ }^{13}$ reflejan esta preocupación por la competencia intelectual en el estudio de la naturaleza humana y no dudan en rebatir a filósofos y otros sabios. Un claro ejemplo de ello lo tenemos al inicio del tratado Sobre la naturaleza del hombre $(H N)$, escrito en la segunda mitad del siglo v a.C. ${ }^{14}$ :

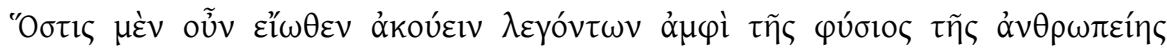

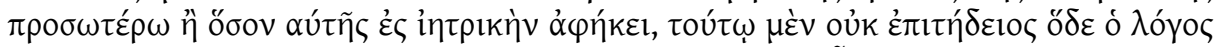

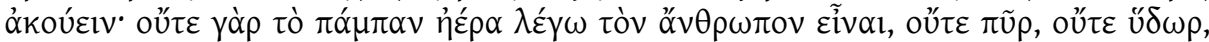

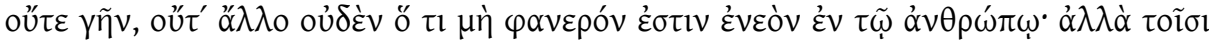

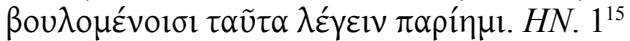

Quien esté habituado a los que hablan acerca de la naturaleza humana en términos que sobrepasan lo estrictamente médico no hallará provecho en esta disertación, pues de ningún modo afirmo que el hombre sea aire, fuego, agua, tierra o cualquier otro elemento que no sea visible en el ser humano, sino que dejo estas ideas a aquel que quiera explicarlas.

Aquellos que no hablaban sobre el ser humano en términos médicos eran los filósofos, en especial Meliso de Samos, nombrado al final del capítulo. Acto seguido el autor del tratado refuta la teoría monista sobre el ser humano, defendida por algunos médicos y la contrapone a su teoría humoral, asegurando que el cuerpo humano estaba compuesto de sangre, pituita, bilis amarilla y bilis negra (HN. 2) ${ }^{16}$. En realidad, el autor buscaba diferenciarse de los filósofos en general y de los médicos monistas,

${ }^{11}$ Heráclito: Arist. Met. 984a; Simpl., Fís. 23, 33; Tales: Arist., Met. 983b; Simpl. Fís. 23, 21-29 y 458, 23-25, Hipól. I. 1, 1 y, finalmente, Anaxímenes: Arist., Met. 984a; Simpl. Fís. 24, 26-28. Las referencias anteriores se hallan compiladas y traducidas en, Eggers y Juliá (1978) y en Longrigg (1998: 31-40). Para una aproximación a esta temática, véase Graham (1999), Sedley (1999) y Dillon (2000: 71-72).

${ }^{12}$ En general, sobre la relación entre filosofía y pensamiento médico véase Longrigg (1993: 24-81) y la reciente publicación del congreso, Hippocrates in Context, en especial Demont (2005) y Le Blay (2005), con bibliografía actualizada.

${ }^{13}$ El Corpus Hipocrático es un conglomerado de tratados teóricos y prácticos de contenido médico que se escribieron entre los siglos v y III a.C. Persisten los debates en torno a la autoría de los diferentes opúsculos aunque, a día de hoy, se impone la idea de que Hipócrates de Cos pudo escribir sólo alguno de ellos, López Férez (2009: 244). Una disertación didáctica sobre los tratados más importantes y la relación que hay entre ellos puede verse en Lloyd (2003: 41-44).

${ }^{14}$ Cano Cuenca (2003: 25).

15 Texto en Jones (1931). Traducción de Cano Cuenca (2003b).

${ }^{16}$ Pigeaud (1996: 778-779) repasa en líneas generales los diferentes sistemas humorales hipocráticos. 
de ahí que la cuestión de fondo sea la autoridad intelectual del médico frente a otras disciplinas y otros colegas. No fue éste un problema menor para médicos itinerantes o médicos públicos que debían realizar una prueba oral de sus conocimientos ante un auditorio si querían ejercer su profesión ${ }^{17}$ (Plat. Gor. 514d). Además, aquellos que conseguían, gracias a sus conocimientos y resultados, obtener la confianza de una ciudad debían renovar el contrato anualmente ${ }^{18}$. Así pues, la argumentación mediante la palabra era esencial para conseguir el trabajo y conservar el estatus social que comportaba la medicina ${ }^{19}$. Buena prueba de la importancia de la palabra para los médicos la encontramos en Platón, Banquete 175a-177d, donde el médico, Erixímaco propone que cada comensal realice un discurso sobre un tema que él había propuesto. También en el tratado hipocrático, Pronóstico 1, se recomendaba que el médico se ejercitara en la previsión o juicio sobre la enfermedad pues, si conseguía relatar los síntomas que el paciente no explicaba, conseguiría una mayor confianza y respeto ${ }^{20}$. Por lo tanto, el médico debía tener facilidad de palabra, al igual que el resto de intelectuales de su época, no sólo para comunicarse o persuadir al paciente, sino para defender su arte en público ${ }^{21}$.

Teniendo en cuenta la necesidad que el médico tenía de prestigio, es preciso poner de manifiesto la importancia de la retórica en la medicina hipocrática así como la función del médico en la transmisión de las ideas sobre la naturaleza del hombre y las distintas formas de restituir la salud. Para ello, repasaremos en primer lugar aquellos textos hipocráticos que pudieron escribirse para ser expuestos oralmente, utilizándose en la formación intelectual de los médicos y, en segundo lugar, analizaremos fuentes literarias y epigráficas que aporten información sobre el médico itinerante y la posible difusión de las ideas médicas.

\section{MEDICINA Y RETÓRICA}

Durante la época arcaica y clásica era usual la transmisión de conocimientos mediante la palabra. Así, conceptos abstractos como el Hades, lo ignoto o lo invisible se transmitían oralmente a través de poemas, tragedias y otros recursos literarios ${ }^{22}$. Los inicios de la historiografía en Grecia también se difundieron de esta forma, hasta la

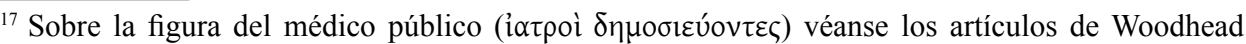
(1952) y Gil (1973) quien, utilizando la comedia como fuente: especialmente Aristófanes Pluto 408ff y un fragmento de Fenícides de Mégara (4 Edm. III A 248), diserta sobre el alcance social de su actividad, su nivel de vida y su salario en la época clásica.

${ }^{18}$ Samama (2003: 39).

${ }^{19}$ Creemos que Laín (1987) ofrece el mejor análisis sobre las técnicas comunicativas del médico hipocrático.

${ }^{20} \mathrm{El}$ respeto era muy importante como vemos en Alcifrón III. 4, donde el médico Acesilao y sus discípulos son ridiculizados por sus prácticas médicas, siendo los dioses quienes finalmente salvan al paciente. Por descontado, los errores médicos eran parodiables, según vemos en la comedia (Gil 1973: 259).

${ }^{21}$ Sobre la defensa de la téchne en estos contextos sociales véase Lara Nava (2004: 46).

${ }^{22}$ Segal (1991: 194-197). 
figura de Tucídides, historiador que entendió la escritura como medio de divulgar el saber ${ }^{23}$. Al margen de la intencionalidad de cada obra, en la historiografía clásica de Heródoto, Tucídides o Jenofonte, encontramos innumerables reproducciones de discursos de todo tipo: ante la Asamblea, ante la tropa o entre personajes relevantes ${ }^{24}$. En general Aristóteles Retórica 1358a y ss, defendía que había tres tipos de discursos: los políticos ante la asamblea del pueblo, los judiciales ante el tribunal y los epidícticos, que buscaban alabar públicamente algún aspecto. Así, la retórica se consideró un arte práctico, como la medicina, que ayudaba a fabricar un discurso con fines persuasivos (judicial y político) o simplemente para deleite del público ${ }^{25}$.

La relación entre medicina y retórica está atestiguada en Platón Gor. 464a y Fedro $270 \mathrm{~b}$, donde el filósofo situó la medicina como paradigma de la verdadera retórica. Siguiendo a Jouanna, creemos que la forma de discernir los tratados del Corpus que fueron escritos con vistas a ser expuestos (epidícticos) es identificando térmi-

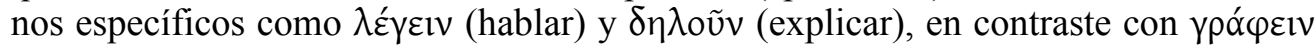
$\left(\right.$ escribir ${ }^{26}$. En nuestra opinión, el tratado Sobre la medicina antigua 1 es una obra fundamental para constatar que la medicina se escribía y se hablaba, ya que comienza

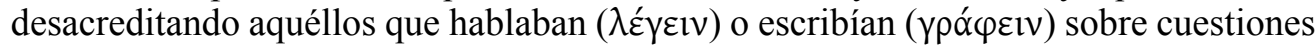
médicas sin tener en cuenta el origen de la enfermedad. En el mismo tratado, Sobre la medicina antigua 2 , se aborda la actitud comunicativa que debe seguir el médico

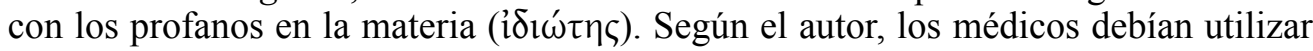
un lenguaje asequible para el público a fin de que el paciente pudiera comprender su dolencia ${ }^{27}$. Esto se aprecia en Sobre la Naturaleza del niño 15, donde el autor relató cómo pudo observar un embrión de seis días. Al parecer, la patrona de un burdel recurrió al autor del tratado para interrumpir el embarazo de una de sus meretrices. Éste, tras escucharla, le indicó que saltara siete veces hasta que los talones tocaran sus nalgas, cayendo el embrión al suelo a la vista de todos. Dejando de lado la veracidad del relato, el pasaje es interesante por dos motivos: en primer lugar porque incide en la importancia del prestigio en la práctica de la medicina y, en segundo lugar, porque detalla un ejemplo claro de la relación comunicativa entre médico y paciente ${ }^{28}$. Estas consideraciones obligaban al médico a poseer una fuerte competencia en materia dialéctica y a un esfuerzo en la labor de comunicación.

El tono divulgador de la medicina adquiere gran importancia en Sobre la ciencia médica, tratado sobre el que se especula con la posibilidad de que hubiera sido escrito

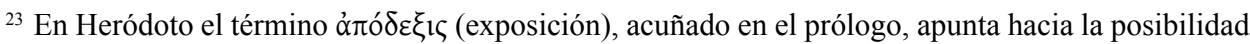
de que la obra se concibiera para ser expuesta en público; Bakker (2002: 4).

${ }^{24}$ En Tucídides este recurso expositivo alcanza una importancia capital, baste recordar la recreación de los diferentes discursos a lo largo de todo el primer libro. Véase Iglesias Zoido (2008) y Sierra (en prensa) y, para el caso de Jenofonte, véase Varias (2010: 677 y ss).

${ }^{25}$ Rodríguez-Alfageme (1997: 153) y Fernández Corte (2010: 171-172).

${ }^{26}$ Jouanna (1984: 29).

${ }^{27}$ Existen muchas más evidencias en este tratado para calificarlo de epidíctico. Véase Jouanna (1990: 9-14), donde se demuestra el carácter retórico de la obra pero se expresan dudas sobre el destinatario del contenido; ¿especialistas o profanos?

${ }^{28}$ Laín (1987: 177) destaca la importancia de la expresión verbal en la génesis de un clima de prestigio alrededor del médico. 
por un sofista ${ }^{29}$. Al inicio de dicho opúsculo se refiere la existencia de ciertos difamadores públicos de las disciplinas basadas en el empirismo y, por esto, el autor inició una exposición razonada para refutarlos (Sobre la ciencia médica 4). Todavía más explícito es el tratado Sobre los vientos, donde el autor comenta lo que sigue:

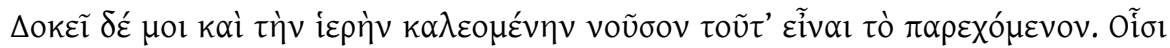

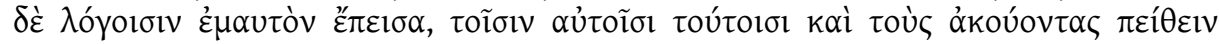
$\pi \varepsilon 1 \rho \eta ́ \sigma o \mu \alpha 1$. Sobre los vientos, $14^{30}$.

En mi opinión, la enfermedad llamada sagrada también está causada por lo mismo. Con las mismas razones que me han convencido intentaré convencer al auditorio.

El destinatario del pasaje anterior era un público interesado en la explicación racional de la denominada «enfermedad sagrada». De igual forma, al inicio del tratado Sobre el arte, también se menciona explícitamente la existencia de discursos en estos ámbitos, por lo que coincidimos con Jouanna en apreciar que ambos tratados deben considerarse como epidícticos ${ }^{31}$. Sin ir más lejos, la enfermedad llamada sagrada fue un tema ampliamente debatido en la Antigüedad. En Sobre la enfermedad sagrada 1, el autor hace una defensa racional del origen de la enfermedad y, al final del capítulo, señala que hablar de los orígenes de todas las enfermedades haría prolija la charla ${ }^{32}$. Acto seguido arremete contra magos, purificadores y otros rivales que declamaban en público sobre esta dolencia. El mismo Heródoto (III. 29) se hizo eco de las opiniones hipocráticas alrededor de esta enfermedad al relacionar la supuesta locura del rey persa Cambises con la denominada «enfermedad sagrada» ${ }^{33}$.

A tenor de los testimonios propuestos y otros que por falta de espacio no abordaremos, entendemos que, para el médico del siglo v a.C., la comunicación verbal era un elemento indispensable. En el correcto desarrollo de su profesión, la fluidez al hablar no sólo era garantía de una defensa de su arte ( $\tau \dot{\chi} \chi \vee \eta)$ sino un elemento de divulgación de ideas como la naturaleza del hombre, la salud, la enfermedad y los métodos de curación $^{34}$. Pero una buena oratoria no sería suficiente para garantizar la difusión de estos conceptos médicos. Es por esto que consideramos oportuno enlazar la vertiente retórica de la medicina con la figura del médico itinerante ${ }^{35}$.

${ }^{29}$ Quizás Hipias de Hélide (García Gual 2000: XI). Los sofistas se mostraron receptivos al conocimiento médico (Rodríguez Alfageme 1997: 155).

${ }^{30}$ Texto original y traducción de Jouanna (1988, CUF). Traducción personal del original francés.

31 Jouanna (1984: 28) y (1988: 10-11 y 173).

32 Sobre el racionalismo hipocrático en la «enfermedad sagrada» véase Joly (1966: 212-213).

33 Sobre la interpretación de la locura en Heródoto véase Dodds (1980: 72).

${ }^{34}$ Lloyd (1991: 137) y Rodríguez Alfageme (1997: 162).

${ }^{35}$ La difusión de ideas es un elemento importante en la configuración del arte médico griego que también recibió fuertes influencias de Oriente Próximo, como ha mostrado recientemente Thomas (2004: 175 y ss). 


\section{EL VIAJE DE LA MEDICINA}

La principal fuente que atestigua la existencia del médico itinerante es el mismo Corpus hippocraticum. En Aires, aguas y lugares 1, se explicitan las medidas que un médico debía tomar al llegar a una ciudad desconocida para la correcta evaluación de la salubridad general del lugar ${ }^{36}$. El dato anterior prueba la movilidad de estos intelectuales que, en cada ciudad, debían demostrar sus habilidades para ser contratados ${ }^{37}$. Estaríamos ante un caso análogo al de los sofistas, que iban por las diferentes poleis griegas ofreciendo sus servicios ${ }^{38}$. Otro tratado, Epidemias, es muy relevante en la percepción de la labor del médico itinerante ${ }^{39}$. Uno de sus autores recopiló sus experiencias como médico en Tasos, recogiendo los cambios de estación, el clima, la orientación de los lugares y la evolución de las enfermedades en este contexto, justo lo que recomendaba al médico el tratado Aires, aguas y lugares cuando llegaba a una ciudad desconocida.

Un testimonio directo, aunque más tardío (s. I-II d.C.), de este tipo de médico lo hallamos en el epitafio dedicado a Aptus, encontrado en Dion, antigua Macedonia:

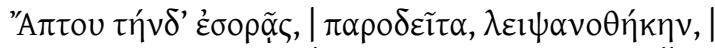

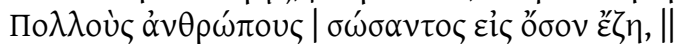

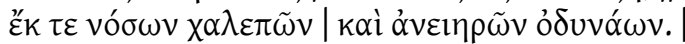

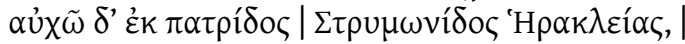

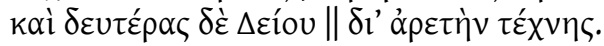

Tú ves aquí, caminante, los restos de Aptus, que en vida salvó a mucha gente de graves enfermedades y amargos dolores. Tuve por primera patria Heraclea de Estrimón y me siento orgulloso de tener a Dion como segunda, gracias al valor de mi arte. ${ }^{40}$

El epígrafe muestra el mismo tipo de profesional que se describe en Aires, aguas $y$ lugares, esto es, un médico que llegaba a una ciudad a ganarse la confianza de una población con palabra y resultados. La epigrafía sigue mostrando ejemplos similares de estos médicos, como el acarnanio Evenor que llegó a ser próxeno de Atenas alrededor del 337 a.C (IG II 242$)$, o Hermias de Cos (s. III a.C), que trabajó en Halicarnaso donde incluso le rindieron honores ${ }^{41}$. El arte médico también llegó a Esparta como muestran los epígrafes $I G$ IV 159, siglo III a.C., donde encontramos a un médico de la familia Nébridas y, un ejemplo más tardío (s. I a.C.), Damias de Lacedemonia $(I G \mathrm{~V} 1,1145$, SEG 37 (1987), 1789) ${ }^{42}$.

${ }^{36} \mathrm{El}$ tratado Aires, aguas y lugares estudia la influencia del medio ambiente, las estaciones de año y el clima sobre el ser humano, una temática análoga a Sobre las Semanas (Joly 1966: 180 y ss). Destaca en el capítulo 12 la comparación entre griegos y asiáticos, con enorme trascendencia sobre la alteridad en la Antigüedad (Jouanna 1996: 8-10 y 54-64).

${ }^{37}$ Véase Rodríguez Alfageme (1997: 152).

${ }^{38}$ Joly (1966: 183), Jouanna (1996: 11).

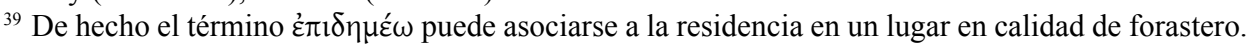

${ }^{40} S E G 31$ (1981), 630; Cormack (1970: 58) y Peek (1971: 185). La traducción francesa corresponde a Samama (2003: 183) y la versión castellana es mía.

${ }^{41}$ Samama (2003: 234-237). Pueden seguirse más ejemplos en este excepcional trabajo.

${ }^{42}$ Textos en Samama (2003: 137 y 141). 
Sin embargo, es en el relato histórico donde aparecen los médicos itinerantes más famosos. Un caso destacable es Democedes de Crotona (segunda mitad del VI a.C.) cuya curiosa historia recogió Heródoto (III. 131). Al parecer, Democedes partió desde su patria natal hacia Egina por desavenencias con su padre. En dicha isla superó en pericia al resto de médicos y consiguió, al segundo año de estancia, que lo admitieran como médico oficial a cambio de un talento anual de plata. Al tercer año los atenienses contrataron sus servicios por cien minas y al cuarto el tirano Polícrates de Samos lo hizo por dos talentos de plata. Finalmente, tras la campaña del sátrapa Oretes contra Polícrates, Democedes acabó como esclavo en la corte del rey Darío I ${ }^{43}$.

Ctesias de Cnido, a finales del siglo v a.C., constituye otro ejemplo del tránsito de los médicos por la geografía griega. Pariente de Hipócrates y miembro de la prestigiosa familia de los asclepíadas, fue capturado por los persas, como Democedes ${ }^{44}$. La presencia de Ctesias como médico de Artajerjes II está documentado en Jenofonte, Anábasis I. 8 y VIII. 6, en Diodoro II. 32, y Plutarco, Artaj. 13. Sin duda este es un caso complejo pues su fama como historiador precede a la de médico, no en vano escribió una Indica y una Persica. Como señala Lenfant, resulta curiosa la influencia de Heródoto en la obra de Ctesias, que retoma algunos episodios abordados por el historiador de Halicarnaso ${ }^{45}$. No obstante, esto prueba el espíritu curioso de este intelectual que, en su cautiverio, no sólo ejercía la medicina sino que también cultivaba otros campos intelectuales.

Por consiguiente, a la luz de estos ejemplos podemos afirmar que tanto la epigrafía como las fuentes escritas atestiguan la actividad de los médicos itinerantes por Grecia. A partir de la época clásica y desde las distintas escuelas, los médicos iban deambulando ciudad por ciudad ofreciendo sus servicios y compitiendo con otros colegas o sabios que trataban de convencer a los ciudadanos de sus conocimientos.

\section{LA DIFUSIÓN DEL CONCEPTO RACIONAL DEL HOMBRE}

Los médicos hipocráticos eran intelectuales reputados en la comprensión de la naturaleza humana y sus dolencias, campo de especial interés para los sabios griegos de época clásica. En este sentido, los médicos entraron en dura competencia por afianzarse entre filósofos y otros intelectuales ${ }^{46}$. Por esto, el dominio de la retórica era esencial pues les daba herramientas para defenderse en los debates públicos sobre la constitución del hombre, la salud y los medios para preservarla. De esta forma, los médicos

${ }^{43}$ No relataremos todas las peripecias de Democedes en la corte persa. Recientemente, Davies (2010) ha discutido en extensión este tema, poniendo en duda la veracidad de la mayoría de vicisitudes que narró Heródoto. Por nuestra parte, nos quedamos con el testimonio que ofrece acerca de la actividad de los médicos itinerantes en Grecia.

${ }^{44}$ Lenfant (2010: 233).

${ }^{45}$ Lenfant (2004: XXVIII). A su vez, la influencia de los escritos hipocráticos en Heródoto está ampliamente estudiada cf. Jouanna (1981), Corcella (1984: 244-250), Dawson (1986), Thomas (2002: 28 y 74), García González (2007: 347-390) y Sierra (en prensa).

${ }^{46}$ Tanto fue así que la medicina se incorporó a la paideia griega (Jaeger 1944: 8 y ss.). 
no sólo debían entender y razonar sobre estas ideas sino que era necesario explicarlas a colegas y profanos. Entonces, no es de extrañar que encontremos evidencias del uso retórico de tratados como Sobre al Arte, Sobre los Vientos o Sobre la medicina antigua, por citar algunos.

Por otro lado, la figura del médico itinerante, atestiguada por la epigrafía, las fuentes médicas escritas y la historia, pudo contribuir de forma decisiva a la proliferación de conceptos como la teoría humoral, la relación entre el medio ambiente, clima y el hombre o la importancia del estilo de vida ( $\left.\delta_{i ́ \alpha} \iota \tau \alpha\right)$ en la conservación de equilibrio natural de la salud. Por ello, concluimos que la vertiente retórica de la medicina, unida a la movilidad de los médicos fueron determinantes en la asimilación del concepto racional sobre el ser humano en época clásica.

\section{BiBLIOGRAFÍA}

BAKker, E. J. (2002), «The making of History: Herodotus' Historiēs Apodexis», en Bakker, E. J.; De Jong, I. J. F.; Van Wess, H. (eds), Brill's Companion to Herodotus, Leiden: Brill: 3-32. Cano Cuenca, J. (2003), «Introducción», en Tratados Hipocráticos VIII, Madrid: Gredos: 13-28.

- (2003b), «Sobre la Naturaleza del hombre», en Tratados Hipocráticos VIII, Madrid: Gredos: 29-63. (traducción)

Corcella, A. (1984), Erodoto e l'analogia, Palermo: Sellerio.

Cormack, J. M. R. (1970), «Inscriptions from Pieria», Klio 52: 49-66.

DAvies, M. (2010), «From Rags to Riches: Democedes of Croton and the Credibility of Herodotus», Bulletin of the Institute of Classical Studies 53 (2): 19-44.

Dawson, W. R. (1986), «Herodotus as a medical writer», Bulletin of the Institute of Classical Studies 33: 87-96.

Demont, P. (2005), «About philosophy and humoural medicine» en, Van der Eijk, Ph. (ed.), Hippocrattes in Context. Papers read at the XIth International Hippocrates Colloquium. University of Newcastle Upon Tyne. 27-31 August 2002. Leiden-Boston: Brill: 271-288.

Dillon, J. (2000), «El ser y las regiones del ser», en Brunschwig, J.; Lloyd, G. (eds), El saber griego: diccionario crítico, Madrid: Akal: 69-84.

Dodds, E. R. (1980), Los griegos y lo irracional, Madrid: Alianza. (or. 1951, The Greeks and the Irrational, Berkeley and Los Angeles: University of California Press)

Eggers, C.; Juliá, V. E. (1978), Los Filósofos Presocráticos I, Madrid: Gredos.

Fernández Corte, J. C. (2010), «Retórica, literatura y eloquentia», en Cortés, F., MéndezDosuna, J. V. (eds), DIC MIHI, MVSA, VIRUM. Homenaje al profesor Antonio López Eire, Salamanca: Universidad de Salamanca: 171-178.

García GonzÁlez, J. A. (2007), Heródoto y la ciencia de su tiempo, Málaga: Universidad de Málaga.

García GuAL, C. (2000), «Introducción General», en Tratados Hipocráticos (Biblioteca Básica Gredos, v.19), Madrid: Gredos (1983): IX-XXXI.

GIL, L. (1973), «Ärztlicher Beistand und attische Komödie. Zur Frage der demosieuontes und Sklaven-Ärzte», Sudhoffs Archiv 57 (3): 255-274. 
Graham, D. W. (1999), «Empedocles and Anaxagoras: Responses to Parmenides», en Long, A. A. (ed), The Cambridge Companion to Early Greek Philosophy, New York: Cambridge University Press: 159-180.

Iglesias Zoido, J. C. (2008), «Tucídides, Historia: los discursos», en Hualde, P., Sanz, M. (eds), La literatura griega y su tradición, Madrid: Akal.

JAEger, W. (1944), Paideia: the ideals of Greek Culture, v. III, New York: Oxford University Press.

Joly, R. (1966), Le Niveau de la Science Hippocratique. Contribution a la Psychologie de l'Histoire des Sciences, Paris: Les Belles Lettres.

- (1967), «Notice», en Hippocrate. Du Régime, Paris: Les Belles Lettres (CUF): IX-XXXIV. Jones, W. H. S. (1931), Hippocrates, vol. I, Cambridge (Mass.)- London.

Jounnna, J. (1981), «Les Causes de la Défaite des Barbares chez Esquile, Hérodote et Hippocrate», Ktema $6: 3-15$.

- (1984), «Rhétorique et Médecine dans la Collection Hippocratique. Contribution à l'Histoire de la Rhétorique au Ve Siècle», REG 97: 26-44.

- (1988), «Notice», en Hippocrate. Des Vents-De l'Art, Paris: Les Belles Lettres (CUF): 9-101.

— (1988b), Des Vents-De l'Art, Paris: Les Belles Lettres (CUF): 102-125. (edición y traducción).

- (1990), «Notice», en Hippocrate. L'Ancienne Médecine, Paris: Les Belles Lettres (CUF): 7-112.

- (1992), «La naissance de la science de l'homme chez les médecins et les savants à l'époque d'Hippocrate: problèmes de méthode», en López Férez, J. A. (ed) Tratados hipocráticos (Estudios acerca de su contenido, forma e influencia). Actas del VII Colloque International Hippocratique, Madrid, 24-29 septiembre de 1990. Madrid: UNED: 91-111.

- (1996), «Notice», en Hippocrate. Airs, Eaux, Lieux, Paris: Les Belles Lettres (CUF): 7-173.

Kosak, J. C. (2004), Heroic Measures. Hippocratic Medicine in the Making of Euripidean Tragedy, Leiden: Brill.

Laín, P. (1970), La Medicina Hipocrática, Madrid: Revista de Occidente.

- (1976), «Estudio preliminar», en Alsina, J., Vintró, E., Sallent, T. (eds), Clásicos de la Medicina Hipocrática, Madrid: CSIC: 9-175.

- (1987), La Curación por la palabra en la Antigüedad Clásica, Barcelona: Anthropos.

LARA NAvA, D. (2004), «El prestigio del médico hipocrático», CFC 14: 45-58.

- (2006), «Praxis y reflexión del médico antiguo», Estudios Clásicos 129: 11-34.

LE BLAY, F. (2005), «Microcosm and macrocosm: the dual direction of analogy in Hippocratic thought and the meteorological tradition», en Van der Eijk, Ph. (ed), Hippocrattes in Context. Papers read at the XIth International Hippocrates Colloquium. University of Newcastle Upon Tyne. 27-31 August 2002. Leiden-Boston: Brill: 251-270.

Lenfant, D. (2004), «Notice», en Ctésias de Cnide. La Perse-L'Inde, Paris: Les Belles Lettres (CUF):VII-CCVII.

- (2010), «Le Medecin Historien», en Zecchini, G. (ed), Lo Storico Antico. Mestieri e figure sociali, Bari: Edipuglia: 231-247.

Lloyd, G. E. R. (1991), Methods and Problems in Greek Science. Selected Papers, Cambridge: Cambridge University Press. 
- (1999), Magic, Reason and Experience. Studies in the Origins and Devolopment of Greek Science, London: Duckworth.

- (2003), In the Grip of Disease. Studies in the Greek imagination, New York: Oxford University Press.

Longrigg, J. (1993), Greek rational Medicine. Philosophy and Medicine from Alcmeon to the Alexandrians, London: Routledge.

- (1998), Greek Medicine. From Heroic to the Hellenistic Age. A Source Book, London: Duckworth.

LóPEZ FÉREZ, J. A. (1986), «Hipócrates y los escritos hipocráticos: origen de la medicina científica», Epos 2: 157-176.

- (2009), «Un pasaje importante para el estudio de los ejercicios físicos en los Tratados médicos Hipocráticos», Humanitas 61: 243-281.

Peek, W. (19719, «Milesische Versinschriften», ZPE 7 (2): 193-226.

Pigeaud, J. (1996), «Il medico e la malattia», en Settis, S. (ed), I Greci. Storia Cultura Arte Società, v.1, Torino: Einaudi: 771-814.

Rodríguez Alfageme, I. (1997), «Retórica, Comedia y Medicina: sobre Ar. Ran. 940-947», en López Eire, A. (ed), Sociedad, Política y Literatura: comedia griega Antigua, Actas del I Congreso Internacional (Salamanca, 1996), Salamanca: Logo:151-172.

Samama, E. (2003), Les Médecins dans le Monde Grec. Sources Épigraphiques sur la Naissance d'un corps Médical, Genève: Droz.

Schiefsky, M. J. (2005), Hippocrates 'On Ancient Medicine', Leiden: Brill.

SEdley, D. (1999), «Parmenides and Melissus», en Long, A. A. (ed), The Cambridge Companion to Early Greek Philosophy, New York: Cambridge University Press: 113-133.

Segal, C. (1991), «L'Auditore e lo Spettatore», en Vernant, J. P. (ed), L’Uomo Greco, Bari: Laterza: $187-218$.

Sierra, C. (a), «El Heródoto Nosológico» (en prensa).

- (b) «La Otra Pentecontecia» (próxima aparición en revista Ágora. Estudos Classicos em debate).

Thomas, R. (2002), Herodotus in Context. Ethnography, Science and the Art of Persuasion, Cambridge: Cambridge University Press.

- (2004), "Greek Medicine and Babylonia Wisdom: Circulation of Knowledge and Channels of Transmission in the Archaic and Classical Periods», en Horstmanshoff, H. F. J., Stol, M. (eds), Magic and Rationality in Ancient Near Eastern and Graeco-Roman Medicine, Leiden: Brill: 175-186.

VARIAS, C. (2010), «Retórica e ideología en los discursos de la Anábasis de Jenofonte: Un caso particular (An. 5. 8. 13-26)», en Cortés, F., Méndez-Dosuna, J. V. (eds), DIC MIHI, MVSA, VIRUM. Homenaje al profesor Antonio López Eire, Salamanca: Universidad de Salamanca: 677-684.

Woodhead, A. G. (1952), «The State Health in Ancient Greece», Cambridge Historical Journal 10 (3): 235-253. 